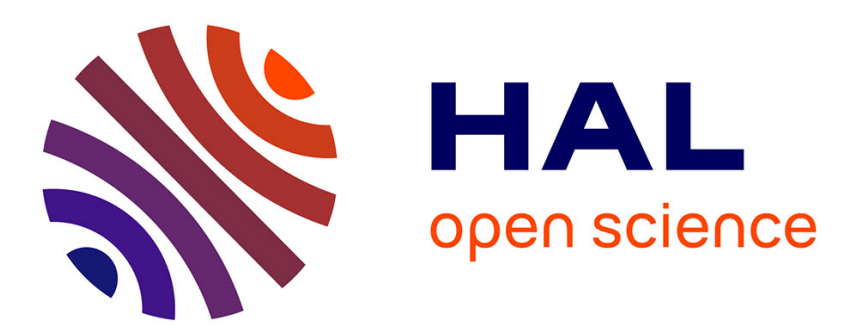

\title{
Limit cycle generation for a class of non-linear systems with jumps using a low dimensional predictive control
}

\author{
Ahmed Chemori, Mazen Alamir
}

\section{To cite this version:}

Ahmed Chemori, Mazen Alamir. Limit cycle generation for a class of non-linear systems with jumps using a low dimensional predictive control. International Journal of Control, 2006, 78 (15), pp.12061217. 10.1080/00207170500268331 . lirmm-02502328

\section{HAL Id: lirmm-02502328 https://hal-lirmm.ccsd.cnrs.fr/lirmm-02502328}

Submitted on 9 Mar 2020

HAL is a multi-disciplinary open access archive for the deposit and dissemination of scientific research documents, whether they are published or not. The documents may come from teaching and research institutions in France or abroad, or from public or private research centers.
L'archive ouverte pluridisciplinaire HAL, est destinée au dépôt et à la diffusion de documents scientifiques de niveau recherche, publiés ou non, émanant des établissements d'enseignement et de recherche français ou étrangers, des laboratoires publics ou privés. 
'Submitted to International Journal of Control'

\title{
Limit cycle generation for a class of nonlinear systems with jumps using a low dimensional predictive control
}

\author{
A. Chemori* and M. Alamir \\ LAG-ENSIEG, CNRS UMR 5528, BP 46 \\ Domaine Universitaire, 38402 Saint Martin d'Hères, France \\ * : e-mail: Ahmed.Chemori@inpg.fr ; Fax: +33 (0)4 76826388
}

\begin{abstract}
In this paper, it is shown that using a low dimensional nonlinear predictive control scheme, provably stable limit cycles can be obtained for open-loop nonlinear systems with unstable equilibrium point. A particular case, where the limit cycle may be reduced to a single point in the state space, can be obtained, which corresponds to asymptotic stabilization. The system may present hybrid nature in the sens that discontinuities (jump phenomena) on the state evolution may be handled. The proposed feedback scheme holds for classical jump-free systems as a particular case. The proposed strategy is illustrated through two examples : a jump-free system (the ball and beam) and a nonlinear hybrid dynamical system including state jumps (the modified impulsive Lorenz chaotic system).
\end{abstract}

Keywords Nonlinear predictive control, zero-dynamics, multi-step limit cycles, Poincaré section, nonlinear hybrid dynamical systems.

\section{Introduction}

Receding horizon control has been first recognized as a powerful approach to stabilize nonlinear systems by [6] for discrete-time nonlinear systems and at roughly the same time by [10] for continuous-time nonlinear systems. Since, lots of works have been done to investigate further results in discrete-time framework [7], robustness [14], discontinuous state feedback formulations [12], free final time formulations [13], real time implementability [15], inverse optimality [8] and hybrid systems related formulations [16]. Beyond the differences in the open-loop optimization problem's formulations, classical formulations share a common feature, namely, the computational difficulty associated to the solution of the open-loop optimization problem. While earlier works used final state equality constraint to insure stability, it is now widely admitted that when optimality is an issue, exact final equality constraints on the state are to be supplanted by a combination of less restrictive inequalities and weighting terms (see for example [3, 14]) giving rise to more robust stabilization results associated to easier computations. However, recent works [2, 9] suggest that when only stabilisation is concerned, formulations with final equalities on the state may lead to fast on-line computations of nonlinear predictive control for a wide class of mechanical under-actuated systems. The present paper falls in this category since it deals with a new low dimensional parameterization of the open-loop control problems associated to predictive control schemes in order to handle situations that may be characterized by the following points:

- First, only stabilization of nonlinear systems is concerned. Namely, the optimization concern is not the main issue. However, problem may concerns point-wise stabilization as well as limit cycles stabilization.

- The proposed illustrative examples of nonlinear systems, may have several equilibrium points (even infinite number of equilibrium points), however, the origin is the equilibrium point under concern.

- The proposed scheme is particularly relevant for under-actuated mechanical systems with a relatively low degree of under-actuation. This is only a practical condition, however, for a theoretical point of 
view, such assumption is not needed, indeed the proposed method is applicable for under-actuated mechanical systems with multi-degrees of under-actuation.

- The proposed scheme is suitable for hybrid systems, namely systems including state jumps for which one aims to establish provably stable limit cycles.

The key tool in the stability analysis for the proposed scheme is the Poincaré section method coupled with the concept of contractive map. In some way, this work is to be connected to recent works on hybrid zero-dynamics [17] in the sense that the parameters of some controlled output is optimized to enhance stability of the remaining dynamics.

The sufficient conditions associated to our formulation (see proposition 1 hereafter) may be checked by means of off-line optimization for a reasonably low dimension of the indirectly controlled sub-state. A graphical intuitive analysis tool may be so obtained (cf figures 3.(a), 6.(a), 9, and ??.(a)).

This paper is organized as follows : in section 2 the problem under consideration is formulated. Some useful definitions and notations are given in section 3 and illustrated on two application examples. The proposed feedback scheme is then explained in section 4. Simulations are proposed in section 5 that concern a jump-free system (the ball \& beam), and a hybrid nonlinear dynamical system with state jumps (the modified Lorenz chaotic system). Finally, the paper ends with a conclusion.

\section{Problem statement}

Consider nonlinear discontinuous dynamical systems [18] given by

$$
\begin{aligned}
\dot{x} & =f(x)+g(x) u & & \text { if } x \notin \mathcal{S}_{0} \\
x\left(t^{+}\right) & =\Delta(x(t)) & & \text { if } x \in \mathcal{S}_{0}
\end{aligned}
$$

where $x \in \mathbb{R}^{n}$ and $u \in \mathbb{R}^{m}$ are the state and the control input respectively, $f, g$ and $\Delta$ are continuous functions in their arguments. Note that equation (2) defines a conditional jump in the state evolution. More precisely, a jump on the state occurs when the state trajectory under (1) hits the "switching surface" defined by

$$
\mathcal{S}_{0}:=\left\{x \in \mathbb{R}^{n} \quad \mid \quad S(x)=0\right\}
$$

where $S: \mathbb{R}^{n} \rightarrow \mathbb{R}^{n_{s}}$ is some continuous map. For the resulting behavior to be well defined under some control-related precautions, the following assumption is used

Assumption 1 There is a set $\mathcal{C} \subset \mathbb{R}^{n}$ such that

$$
\left\{x \in \mathcal{C} \cap \mathcal{S}_{0}\right\} \Rightarrow\{S(\Delta(x)) \geq \gamma(\mathcal{C})>0\}
$$

where $\gamma(\cdot)$ is a strictly positive function defined on subsets of $\mathbb{R}^{n}$ with values in $\mathbb{R}^{n_{s}}$.

This assumption guarantees that the jump takes the state far from the switching surface $\mathcal{S}_{0}$. This with some rather classical continuity and boundedness arguments clearly enables a free-chattering well defined dynamics to take place. The following lemma is a key result when applying the general scheme to classical jump-free systems

LEMMA 1 Any classical $n$-dimensional jump-free system $\dot{x}=f(x)+g(x) u$ may be embedded in an $(n+1)$ dimensional system that has the general form (1)-(2) for which assumption 1 is trivially satisfied.

Proof Let $\tau_{c}>0$ be any given positive real. define the extended $(n+1)$-dimensional state and $(m+1)$ dimensional control

$$
x^{e}:=\left(\begin{array}{c}
x \\
x_{n+1}^{e}
\end{array}\right) \quad ; \quad u^{e}:=\left(\begin{array}{c}
u \\
u_{m+1}^{e}
\end{array}\right)
$$


with the extended dynamics

$$
\dot{x}^{e}=\left(\begin{array}{c}
f(x)+g(x) u \\
u_{m+1}^{e}
\end{array}\right)=:\left(\begin{array}{c}
f(x) \\
0
\end{array}\right)+\left(\begin{array}{cc}
g(x) & 0 \\
0 & 1
\end{array}\right)\left(\begin{array}{c}
u \\
u_{m+1}^{e}
\end{array}\right)=f^{e}\left(x^{e}\right)+g^{e}\left(x^{e}\right) u^{e}
$$

consider the switching surface and the jump functions defined by

$$
S\left(x^{e}\right)=\tau_{c}-x_{n+1}^{e} \quad ; \quad \Delta\left(x^{e}\right)=\left(\begin{array}{l}
x \\
0
\end{array}\right)
$$

with the above notations, it is clear that assumption 1 holds with $\mathcal{C}:=\mathbb{R}^{n+1}$ and $\gamma(\mathcal{C})=\tau_{c}$.

Note that according to lemma 1, an extended system (4) can be defined for any classical jump-free system, once a choice of $\tau_{c}>0$ is made. Throughout the paper such an extended system is called $\tau_{c}$-extended version of the original system.

The aim of this paper is to propose a feedback law that stabilizes the closed-loop trajectory around some limit cyclic bounded trajectory. More precisely, let $\tau_{c}>0$ be some suitable period. Our aim is to prove that under the proposed feedback, there is an integer $k_{0} \in \mathbb{N}$ and bounded trajectory $x_{l}(\cdot)$ defined on $\left[0, k_{0} \tau_{c}^{-}\right]$that is attractive w.r.t the closed loop system's dynamic, namely

$$
\lim _{j \rightarrow \infty} \sup _{\tau \in\left[0, k_{0} \tau_{c}\right]}\left\|x_{c l}\left(j k_{0} \tau_{c}+\tau ; x_{0}\right)-x_{l}(\tau)\right\|=0
$$

where $x_{c l}\left(\cdot ; x_{0}\right)$ is the closed loop system's trajectory starting from the initial condition $\left(0, x_{0}\right)$. The target trajectory $x_{l}$ satisfies the following boundary conditions that are compatible with the jump map $\Delta(\cdot)$ defined by (2), namely

$$
x_{l}\left(k_{0} \tau_{c}^{-}\right)=x^{f} \quad ; \quad x_{l}(0)=\Delta\left(x^{f}\right)
$$

where $x^{f} \in \mathbb{R}^{n}$ is some fixed desired value.

In the case where (5) may not hold globally, we look for a deterministic computable way to evaluate a bounded region of attraction $\mathbb{X}$ such that (5) is satisfied for all $x_{0} \in \mathbb{X}$.

Remark 1 Roughly speaking, the target limit trajectory is defined over an interval of length $k_{0} \tau_{c}$ at the end of which a jump (that may be virtual) occurs. (As a matter of fact, a jump occurs each $\tau_{c}$ time unit as explained later on).

\section{Some definitions \& notations}

In the forthcoming, some basic definitions and notations, used in the remainder of the paper are introduced and elucidated on two illustrative applications that are later used to state the effectiveness of the proposed feedback strategy.

\subsection{Partial feedback linearization}

In the following, the principle of partial feedback liearization [5] is introduced, and illustrated on single-input single-output systems (without loss of generality), which can easily be extended to nonlinear multi-input multi-output systems. Assume that an output map $h: \mathbb{R}^{n} \rightarrow \mathbb{R}^{m}$ is defined, consider the nonlinear system

$$
\begin{aligned}
\dot{x} & =f(x)+g(x) u \\
y & =h(x)
\end{aligned}
$$

let $L_{f} h$ be the lie derivative of $h$, defied by $L_{f} h(x)=\frac{\partial h}{\partial x} \dot{x}=\frac{\partial h}{\partial x}(f(x)+g(x) u)$. The system (7)-(8) has a relative degree $r$ if $\forall x \in \mathbb{R}^{n}$

$$
\left\{\begin{array}{l}
L_{g} L_{f}^{i} h(x)=0 \quad ; \quad \forall i<r-1 \\
L_{g} L_{f}^{r-1} h(x) \neq 0
\end{array}\right.
$$


if $r=n$, input-state linearization is obtained, otherwise (i.e $r<n$ ) partial feedback linearisation is performed, for that, consider the following coordinate transformation $\left(\begin{array}{l}\xi \\ \eta\end{array}\right)=\Phi(x)$ defined by

$$
\left\{\begin{array}{l}
\xi=\left[\begin{array}{llll}
h(x) & L_{f} h(x) & \ldots & L_{f}^{r-1} h(x)
\end{array}\right]^{T} \\
\eta=\left[\begin{array}{llll}
\eta_{1}(x) & \eta_{2}(x) & \ldots & \eta_{n-r}(x)
\end{array}\right]^{T}
\end{array}\right.
$$

where $\eta_{i}$ are independent functions such that $L_{g} \eta_{i}=0 \quad ; \quad \forall x \in \mathbb{R}^{n}$

then $\Phi(x)$ is a diffeomorphism, and we get normal form for a nonlinear system of relative degree $r$

$$
\left\{\begin{array}{l}
\dot{\xi}_{1}=\xi_{2} \\
\vdots \\
\dot{\xi}_{r-1}=\xi_{r} \\
\dot{\xi}_{r}=L_{f}^{r} h(x)+L_{g} L_{f}^{r-1} h(x) u \quad ; \quad x=\Phi^{-1}\left(\begin{array}{l}
\xi \\
\eta
\end{array}\right) \\
\dot{\eta}=Z(\xi, \eta)
\end{array}\right.
$$

Consider now the nonlinear input control $u$ defined by

$$
u=\alpha(x)+\beta(x) v \quad ; \quad \alpha(x)=-\frac{L_{f}^{r} h(x)}{L_{g} L_{f}^{r-1} h(x)} \quad ; \quad \beta(x)=\frac{1}{L_{g} L_{f}^{r-1} h(x)}
$$

which replaced in (11) gives the system dynamic in the new coordinates

$$
\begin{aligned}
& \dot{\xi}=A \xi+B v \quad ; \quad \xi \in \mathbb{R}^{n_{\xi}} \\
& \dot{\eta}=Z(\xi, \eta) \quad ; \quad \eta \in \mathbb{R}^{n_{\eta}}
\end{aligned}
$$

where the pair $(A, B)$ is controllable, $n_{\xi}=r$, and $n_{\eta}=(n-r)$.

It goes without saying that the choice of the output $y=h(x)$ is not unique. Among all possible choices, those leading to a maximal value of $n_{\xi}$ are to be naturally preferred. In the context of systems with jumps and in order for the proposed feedback strategy to be applied, the choice of the output $y=h(x)$ has to meet the following requirement

DeFinition 1 An output map $h: \mathbb{R}^{n} \rightarrow \mathbb{R}^{m}$ is said to be compatible with the dynamics given by (1)-(2) if the following conditions hold

1. There is a map $\bar{S}: \mathbb{R}^{n_{\xi}} \rightarrow \mathbb{R}^{n_{s}}$ such that the following implication holds

$$
\{\bar{S}(\xi)=0\} \Rightarrow\left\{S \circ \Phi^{-1}(\xi, \eta)=0 \text { for all } \eta \in \mathbb{R}^{n_{\eta}}\right\}
$$

2. There is a subset $\overline{\mathcal{C}} \subset \mathbb{R}^{n_{\xi}}$ such that

$$
\{\xi \in \overline{\mathcal{C}}\} \Rightarrow\left\{\Phi^{-1}(\xi, \eta) \in \mathcal{C} \quad \text { for all } \eta \in \mathbb{R}^{n_{\eta}}\right\}
$$

where $\mathcal{C} \subset \mathbb{R}^{n}$ is the set defined in assumption 1 .

Roughly speaking, a compatible output map (if any) leads to a system's state decomposition in which one may control via the linear controllable sub-system (12) both the occurrence of the jump [condition (14)] and the quality (chattering-free) of the resulting behaviour [condition (15)].

Again, the following lemma shows that when a classical system is extended according to lemma 1 to yield a jump system, the resulting $\tau_{c}$-extended system always admits an output map that is compatible with its dynamics. 
LEMMA 2 Any $\tau_{c}$-extended system in the sense of lemma 1 admits a compatible output map.

Proof Take the output map $h^{e}\left(x^{e}\right)=x_{n+1}^{e}$, for this system, the choice $\xi=x_{n+1}^{e}$ is clearly valid. Now since the switching map $S\left(x^{e}\right)=\tau_{c}-x_{n+1}^{e}$ involves only $x_{n+1}^{e}=\xi$, the first point of definition 1 clearly follows with the definition $\bar{S}(\xi)=\tau_{c}-\xi$. The second point comes from the fact that $\mathcal{C}=\mathbb{R}^{n+1}$ and therefore (15) contains no additional requirements. Note that in the case where the original system admits a natural output map $h(x)$ leading to partial linearization by feedback, a natural choice of the extended output map would be $h^{e}\left(x^{e}\right)=\left(\begin{array}{l}h(x) \\ x_{n+1}^{e}\end{array}\right)$ for which the whole argumentation developed above still holds.

\section{$3.2 \quad$ Illustrative Examples}

Before going further, let us illustrate the notations presented above by introducing the following two examples used later to attest the efficiency of the proposed feedback strategy.

\subsubsection{The ball and beam : a classical jump free system}

The system in question is one of the most enduringly popular plants. Depicted in figure 1, it consists of a horizontal beam (with moment of inertia $J$ ) whose angle is controlled by a servo motor (with torque $\tau$ ). A rolling ball (of mass $m$, radius $R$, and moment of inertia $J_{b}$ ) is placed on the beam where it is allowed to roll with one degree of freedom along the length of the beam. As the beam turns by an angle $\theta$, the gravity causes the ball to roll along the beam.

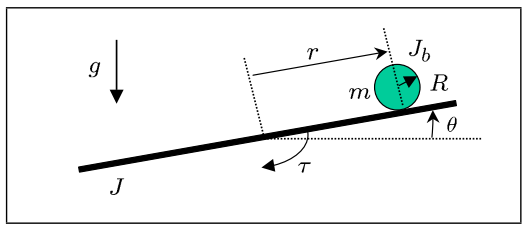

Figure 1: The ball and beam plant

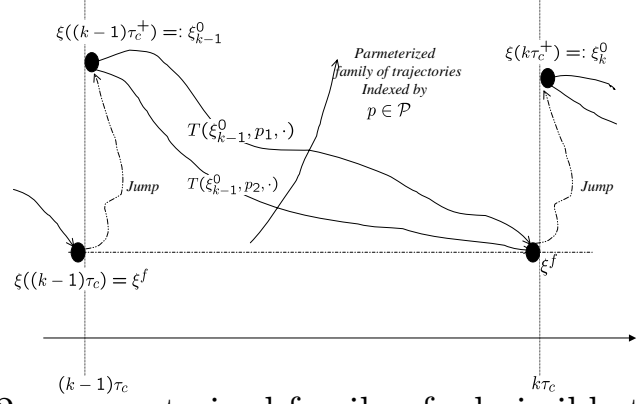

Figure 2: parameterized family of admissible trajectories

The dynamic model [4] describing this system is given by the following equations

$$
\left\{\begin{array}{l}
\left(m r^{2}+J+J_{b}\right) \ddot{\theta}+2 m r \dot{r} \dot{\theta}+m g r \cos \theta=u \\
\ddot{r}-B r \theta \dot{\theta}^{2}+B g \sin \theta=0
\end{array} \quad ; \text { where } B=\frac{m}{\frac{J_{b}}{R^{2}}+m}\right.
$$

using the following notation $\quad x=\left(\begin{array}{llll}\theta & r & \dot{\theta} & \dot{r}\end{array}\right)^{T} \in \mathbb{R}^{4} \quad$ the system model (16) takes the form (7), with the following definition of the functions $f$, and $g$

$$
f(x)=\left(\begin{array}{c}
x_{3} \\
x_{4} \\
-\frac{1}{\left(m x_{2}^{2}+J+J_{b}\right)}\left(2 m x_{2} x_{3} x_{4}+m g x_{2} \cos x_{1}\right) \\
B x_{1} x_{2} x_{3}^{2}-B g \sin x_{1}
\end{array}\right) \quad ; \quad g(x)=\left(\begin{array}{c}
0 \\
0 \\
\frac{1}{m x_{2}^{2}+J+J_{b}} \\
0
\end{array}\right)
$$

the origin is an equilibrium point unstable for the open-loop system. Again, by application of lemma 1, a $\tau_{c}$-extended version of the original system is obtained. An output compatible map is constructed with $h(x)=\theta$ leading to

$$
\xi=\left(\begin{array}{lll}
\theta & \dot{\theta} & x_{5}^{e}
\end{array}\right)^{T} \in \mathbb{R}^{3} \quad ; \quad \eta=\left(\begin{array}{ll}
r & \dot{r}
\end{array}\right)^{T} \in \mathbb{R}^{2}
$$

where $x_{5}^{e}$ is the additional state invoked in lemma 2 . 


\subsubsection{The modified Impulsive Lorenz chaotic dynamical system : a system with state jumps}

Consider the modified Lorenz system which is a benchmark of chaotic systems described by the following equations ${ }^{1}$ :

$$
\left\{\begin{array}{l}
\dot{x}_{1}(t)=-\sigma x_{1}(t)+\sigma x_{2}(t) \\
\dot{x}_{2}(t)=r x_{1}(t)-x_{2}(t)-x_{1}(t) x_{3}(t)+u \quad ; \quad \text { if } \quad t \neq k \tau_{c} \\
\dot{x}_{3}(t)=x_{1}(t) x_{2}(t)-b x_{3}(t)
\end{array}\right.
$$

together with

$$
\left(\begin{array}{l}
x_{1}\left(t^{+}\right) \\
x_{2}\left(t^{+}\right) \\
x_{3}\left(t^{+}\right)
\end{array}\right)=\Delta\left(\begin{array}{l}
x_{1}(t) \\
x_{2}(t) \\
x_{3}(t)
\end{array}\right)=(I+B)\left(\begin{array}{l}
x_{1}(t) \\
x_{2}(t) \\
x_{3}(t)
\end{array}\right) \quad ; \quad \text { if } \quad t=k \tau_{c}
$$

where $\sigma, r, b$ are three positive parameters, $I$ is the identity matrix, and the matrix $B$ is given by

$$
B=\left(\begin{array}{ccc}
\mu & 0 & 0 \\
0 & -0.6 & 0 \\
0 & 0 & -0.6
\end{array}\right)
$$

when the following choice $\sigma=10, \quad r=28, \quad b=8 / 3 \quad$ is made, the autonomous control-free Lorenz system is known to be chaotic [18]. Using the extended state vector $x=\left(\begin{array}{llll}x_{1} & x_{2} & x_{3} & x_{4}\end{array}\right)^{T}$, with $\dot{x}_{4}=1$ and the extended jump function

$$
\left.\left(\begin{array}{l}
x_{1}\left(t^{+}\right) \\
x_{2}\left(t^{+}\right) \\
x_{3}\left(t^{+}\right) \\
x_{4}\left(t^{+}\right)
\end{array}\right)=\left(\begin{array}{c}
x_{1} \\
x_{2} \\
x_{3}
\end{array}\right)\right) ; t=k \tau_{c}
$$

the system takes the form (1)-(2) With

$$
f(x)=\left(\begin{array}{c}
-\sigma x_{1}+\sigma x_{2} \\
r x_{1}-x_{2}-x_{1} x_{3} \\
x_{1} x_{2}-b x_{3} \\
1
\end{array}\right) \quad ; \quad g(x)=\left(\begin{array}{l}
0 \\
1 \\
0 \\
0
\end{array}\right)
$$

And the switching surface is defined by

$$
S(x)=x_{4}-\tau_{c}
$$

Using the coordinate transformation (c.f sect. (3.1)), the following choice of the output map

$$
h(x):=\left(x_{2}, x_{4}\right)^{T} \in \mathbb{R}^{2}
$$

leads to the following definition of the sub-states $(\xi, \eta)$

$$
\xi:=\left(x_{2}, x_{4}\right)^{T} \in \mathbb{R}^{2} \quad ; \quad \eta:=\left(\begin{array}{ll}
x_{1} & x_{3}
\end{array}\right)^{T} \in \mathbb{R}^{2}
$$

Furthermore, the output map (21) is compatible with the dynamics (18)-(19) in the sense of definition 1, since its two conditions are satisfied.

\footnotetext{
${ }^{1}$ note that the original autonomous system given in [18], has been modified by introducing a control input.
} 


\section{The proposed feedback scheme}

Let some fixed period $\tau_{c}>0$ be chosen. The idea is to use the linearly controlled sub-state $\xi$ to periodically hit the switching surface at some pre-determined value $\xi^{f} \in \bar{S}^{-1}(0)$ reflecting the control objective. This is possible provided that the output map $h(x)$ used to perform the partial feedback linearization is compatible with the dynamics (1)-(2) in the sense of definition 1. In this case, one obtains just before the jumps that periodically occurs at instants $t_{k}=k \tau_{c}, k \in \mathbb{N}_{+}$.

$$
\forall k \in \mathbb{N}_{+} \quad: \quad \xi\left(t_{k}^{-}\right)=\xi^{f} \quad ; \quad \bar{S}\left(\xi^{f}\right)=0
$$

It goes without saying that once the pair $\left(\tau_{c}, \xi^{f}\right)$ is given, there is infinitely many ways to realize the end-point condition (22). This is because there are infinitely many trajectories that start at the post jump value $\xi\left(t_{k-1}^{+}\right)$to join the just-before next-jump desired state $\xi\left(t_{k}^{-}\right)=\xi^{f}$ (see Figure 2). That is the reason why the following definition is introduced (see also Figure 2)

Definition 2 Let $n_{p} \in \mathbb{N}$ be some integer. For any subset $\mathcal{P} \subset \mathbb{R}^{n_{p}}$, we call a $\mathcal{P}$-family of admissible parameterized trajectories a map

$$
T: \mathbb{R}^{n_{\xi}} \times \mathcal{P} \times\left[0, \tau_{c}\right] \rightarrow \mathbb{R}^{n_{\xi}}
$$

such that the following conditions are satisfied for all $p \in \mathcal{P}$ and all $\xi^{0} \in \mathbb{R}^{n_{\xi}}$

1. $T\left(\xi^{0}, p, 0\right)=\xi_{0}$ and $T\left(\xi^{0}, p, \tau_{c}\right)=\xi^{f}$

2. $T\left(\xi^{0}, p, \cdot\right)$ is compatible with the linear system (12). That is, if the system (12) is at the initial condition $\left(0, \xi^{0}\right)$, then exact tracking of the trajectory $T\left(\xi^{0}, p, \cdot\right)$ is possible under some suitable timevarying feedback defined over $\left[0, \tau_{c}\right]$ by

$$
v(\tau)=\mathcal{V}_{\left(\xi^{0}, p\right)}(\xi(\tau), \tau) \quad \forall \tau \in\left[0, \tau_{c}\right]
$$

Roughly speaking, a $\mathcal{P}$-family of admissible trajectories provides a finite dimensional parameterization of trajectories joining two points in $\mathbb{R}^{n_{\xi}}$ and that can be exactly tracked by the $\xi$-linear controllable system (12) using the state feedback control (24).

When such a feedback is applied between two jumps, one systematically has $\xi\left(t_{k}^{-}\right)=\xi^{f}$ just before jumps. This make the before jump instants $t_{k}^{-}$a suitable choice to define a Poincaré-section that may be used to study the stability of the overall dynamics. More precisely, if it can be proven that under some suitable choices, the sequence $\left(\eta\left(t_{k}^{-}\right)\right)_{k \in \mathbb{N}_{+}}$obeys an asymptotically stable dynamic law, the stability of the whole system follows. The key feature is that under (24) and for a given fixed value of $\xi^{f}$, there is some $F: \mathbb{R}^{n_{\eta}} \times \mathcal{P} \rightarrow \mathbb{R}^{n_{\eta}}$ such that the dynamic of $\eta$ is given by

$$
\eta\left(t_{k+1}^{-}\right)=F\left(\eta\left(t_{k}^{-}\right), p\left(t_{k}^{+}\right), \xi^{f}\right)
$$

where $p\left(t_{k}^{+}\right) \in \mathcal{P}$ is the parameter that select the trajectory ${ }^{2} T\left(\xi\left(t_{k}^{+}\right), p\left(t_{k}^{+}\right), \cdot\right)$ to be tracked by the sub-state $\xi$ over $\left[t_{k}, t_{k+1}\right]$. Indeed, the state just after the jump that occurs at instant $k$ is given by

$$
\left(\begin{array}{c}
\xi \\
\eta
\end{array}\right)\left(t_{k}^{+}\right)=\Delta\left(\left(\begin{array}{c}
\xi^{f} \\
\eta\left(t_{k}^{-}\right)
\end{array}\right)\right)=:\left(\begin{array}{c}
\xi^{+}\left(\eta\left(t_{k}^{-}\right)\right) \\
\eta^{+}\left(\eta\left(t_{k}^{-}\right)\right)
\end{array}\right)
$$

Furthermore, since the feedback law (24) enables a perfect tracking to be performed over $\left[t_{k}, t_{k+1}\right]$, the predicted evolution of the sub-state $\eta$ over this interval is given by (13), namely

$$
\dot{\eta}(t)=Z\left(\xi^{r e f}(t), \eta(t), v^{r e f}(t)\right)
$$

\footnotetext{
${ }^{2}$ within the $\mathcal{P}$-family of admissible trajectories in the sense of definition 2
} 
where

$$
\begin{aligned}
\xi^{r e f}(t) & =T\left(\xi^{+}\left(\eta\left(t_{k}^{-}\right)\right), p\left(t_{k}^{+}\right), t\right) \\
v^{r e f}(t) & =\mathcal{V}_{\left(\xi^{+}\left(\eta\left(t_{k}^{-}\right)\right), p\left(t_{k}^{+}\right)\right)}\left(\xi^{r e f}(t), t\right)
\end{aligned}
$$

that may be written in the following more compact form

$$
\dot{\eta}(t)=: \varphi\left(t, \eta(t), \eta\left(t_{k}^{-}\right), p\left(t_{k}^{+}\right), \xi^{f}\right) \quad ; \quad t \in\left[t_{k}, t_{k+1}\right]
$$

that gives $(25)$ by integration over $\left[t_{k}, t_{k+1}\right]$ starting from the initial condition $\eta^{+}\left(\eta\left(t_{k}\right)\right)$. Let's summarize LEMMA 3 Assume that assumption 1 holds and that a compatible output map $h(x)$ has been defined (see definition 1) leading to a partial feedback linearization. Let a pair $\left(\tau_{c}, \xi^{f}\right) \in \mathbb{R}_{+} \times \bar{S}^{-1}(0)$ be given. Let $\left(p_{k}\right)_{k \in \mathbb{N}} \in \mathcal{P}^{\mathbb{N}}$ be a sequence of parameters in $\mathcal{P}$.

There is a map $F: \mathbb{R}^{n_{\eta}} \times \mathcal{P} \rightarrow \mathbb{R}^{n_{\eta}}$ such that under the feedback law given by

$$
\left.\left.v(t)=\mathcal{V}_{\left(\xi\left(t_{k}^{+}\right), p_{k}\right)}(\xi(t), t) \quad ; \quad t \in\right] t_{k}, t_{k+1}\right]
$$

the resulting discrete dynamics of $\eta$, which has no finit escape time is given by

$$
\eta\left(t_{k+1}^{-}\right)=F\left(\eta\left(t_{k}^{-}\right), p_{k}, \xi^{f}\right)
$$

Note that (29) is a discrete-time controlled system in which $\eta$ is the state and $p$ is the control input. Therefore, the choice of the control $p_{k}$ may be made by using classical design methodologies for discretetime nonlinear systems. unfortunately, the implicit nature of the map $F(\cdot)$ makes difficult the use of analytical design method and incites to use predictive control tools $[6,1,11]$. Indeed, such strategies need no analytical manipulations to be performed in order to derive the feedback law. More precisely, the control $p_{k}$ is computed by solving the following optimization problem

$$
p_{k}=\hat{p}\left(\eta\left(t_{k}^{-}\right), \xi^{f}, \eta^{f}\right):=\operatorname{Arg} \min _{p \in \mathcal{P}}\left\|F\left(\eta\left(t_{k}^{-}\right), p, \xi^{f}\right)-\eta^{f}\right\|_{Q}^{2}
$$

where $\xi^{f}$ and $\eta^{f}$ are defined by the desired target $x^{f}$ by the coordinates transformation $\Phi$ given by (10), namely

$$
\left(\begin{array}{c}
\xi^{f} \\
\eta^{f}
\end{array}\right):=\Phi\left(x^{f}\right)
$$

The solution of the optimization problem (30) is based on the knowledge of $F\left(\eta\left(t_{k}^{-}\right), p_{k}, \xi^{f}\right)=\eta\left(t_{k+1}^{-}\right)$ which is obtained by prediction, therefore the control approach is a predictive control scheme.

Injecting the feedback (30) in (29) results in the following closed-loop dynamic

$$
\begin{aligned}
\eta\left(t_{k+1}^{-}\right) & =F_{c l}\left(\eta\left(t_{k}^{-}\right), x^{f}\right) \\
& :=F\left(\eta\left(t_{k}^{-}\right), \hat{p}\left(\eta\left(t_{k}^{-}\right), \xi^{f}, \eta^{f}\right), \xi^{f}\right)
\end{aligned}
$$

That can be expressed in a multi-steps form by successive compositions as follows

$$
\eta\left(t_{k+k_{0}}^{-}\right)=F_{c l}^{k_{0}}\left(\eta\left(t_{k}^{-}\right), x^{f}\right)
$$

that is crucial to express the following stability results:

Proposition 1 1. If for some $k_{0} \in \mathbb{N}, Q>0$ and some $\rho>0$, the map $F_{c l}^{k_{0}}$ satisfies the following inequality

$$
\forall r \in\left[0, \rho\left[\quad ; \quad \Psi_{k_{0}}^{Q}(r):=\left[\sup _{\left\|\eta-\eta^{f}\right\|_{Q}^{2}=r}\left\|F_{c l}^{k_{0}}\left(\eta, x^{f}\right)-\eta^{f}\right\|_{Q}^{2}\right] \leq \mu \cdot r \quad ; \quad \mu<1\right.\right.
$$

then the closed-loop trajectory converges to a limit-cycle of length $k_{0}$, that is

$$
\lim _{j \rightarrow \infty} x_{c l}\left(j k_{0}^{-}\right)=x^{f}
$$

for all initial conditions $\xi\left(0^{-}\right)=\xi^{f}$ and $\eta\left(0^{-}\right) \in B\left(\eta^{f}, \rho\right)$. 
2. If for some $k_{0} \in \mathbb{N}$ and $Q>0$, there is some $\rho>0$ and some positive $\varepsilon<\rho$ such that the two following inequalities hold

$$
\sup _{r \leq \varepsilon}\left[\Psi_{k_{0}}^{Q}(r)\right] \leq \varepsilon
$$

and

$$
\forall r \in] \varepsilon, \rho] \quad \Psi_{k_{0}}^{Q}(r) \leq \mu r \quad ; \quad \mu<1
$$

then the set $\mathbb{M}_{\varepsilon}$ defined by

$$
\mathbb{M}_{\varepsilon}:=\left\{\left(\begin{array}{c}
\xi^{f} \\
\eta
\end{array}\right) \quad \mid \quad\left\|\eta-\eta^{f}\right\|_{Q}^{2} \leq \varepsilon\right\}
$$

is invariant and attractive for the poincaré map (33) for all initial condition belonging to the set $\mathbb{M}_{\rho}$ defined by

$$
\mathbb{M}_{\rho}:=\left\{\left(\begin{array}{c}
\xi^{f} \\
\eta
\end{array}\right) \quad \mid \quad\left\|\eta-\eta^{f}\right\|_{Q}^{2} \leq \rho\right\}
$$

namely, starting in $\mathbb{M}_{\rho}$, the poincaré sequence satisfies

$$
\lim _{j \rightarrow \infty}\left(\begin{array}{c}
\xi\left(j k_{0} \tau_{c}^{-}\right) \\
\eta\left(j k_{0} \tau_{c}^{-}\right)
\end{array}\right) \in \mathbb{M}_{\varepsilon}
$$

\section{Proof}

1. Straightforward since under (34), the Poincaré map is contractive and admits $\eta^{f}$ as fixed point provided that the initial condition is such that (34) holds.

2. Under (36), $\mathbb{M}_{\varepsilon}$ is clearly invariant. Furthermore, starting in $\mathbb{M}_{\rho}$, the inequality (37) shows that as long as the image of the Poincaré map remain outside $\mathbb{M}_{\varepsilon}$, one necessarily has

$$
\left\|\eta\left(t_{j k+k_{0}}\right)-\eta^{f}\right\|_{Q}^{2} \leq \mu\left\|\eta\left(t_{j k}\right)-\eta^{f}\right\|_{Q}^{2}
$$

this shows that $\mathbb{M}_{\varepsilon}$ is attractive.

Remark 2 1. Note that the use of the multi-steps map $F^{k_{0}}(\cdot)$ is a crucial feature in the proof of the stability of the resulting closed-loop system. However, the on-line optimization problem that defines the feedback being applied is still based on one-step computation.

2. It is important to underline that the sufficient condition for stability can be checked by off-line computations that amount to compute

$$
\Psi_{k_{0}}^{Q}(r):=\sup _{\left\|\eta-\eta^{f}\right\|_{Q}^{2}=r}\left\|F_{c l}^{k_{0}}\left(\eta, x^{f}\right)-\eta^{f}\right\|_{Q}^{2}
$$

for increasing values of $r$. The fact that such computations may be practically done comes from the fact that for many mechanical system, the dimension $n_{\eta}$ of the sub-state $\eta$ (the zero-dynamic) is relatively low with respect to the whole system state dimension $n$. As an example, for the walking robot, $n_{\eta}=2$ while $n=10$ while for the ball and beam, these dimensions are respectively $n_{\eta}=2$ and $n=4$.

3. Note also that the set of initial conditions leading to a stable closed-loop trajectories is generally much larger than the region of attraction predicted by proposition 1 since the latter is based on the worst case computation (41). 


\begin{tabular}{|c|c|c|}
\hline Parameter & Significance & Value \\
\hline \hline$m$ & mass of the ball & $0.11 \mathrm{~kg}$ \\
\hline$R$ & radius of the ball & $0.015 \mathrm{~m}$ \\
\hline$J_{b}$ & ball's moment of inertia & $9.9910^{-6} \mathrm{~kg} \cdot \mathrm{m}^{2}$ \\
\hline$r$ & the ball position coordinate & variable \\
\hline$L$ & length of the beam & $1.0 \mathrm{~m}$ \\
\hline$J$ & the beam's moment of inertia & $0.125 \mathrm{~kg} \cdot \mathrm{m}^{2}$ \\
\hline$\theta$ & the beam angle coordinate & variable \\
\hline$g$ & the gravitational acceleration & $9.8 \mathrm{~m} . \mathrm{S}^{-} 2$ \\
\hline
\end{tabular}

Table 1: Model parameters values for the ball \& beam system

4. It is important to emphasize that it is only the impact of the closed-loop trajectory with the Poincaré's section that converges to the $x_{f}$ (case of point 1 . of proposition 1) or to the neighborhood $\mathbb{M}_{\varepsilon}$ (case of point 2. of proposition 1). The behavior of the overall trajectory depends on the resulting system's trajectory under the initial condition $x_{f}$.

It is worth noting that in the two examples, the dimension of the on-line optimization problem (dimension of $p$ ) is scalar. This makes easier real-time implementation of the scheme for many quite rapid systems that have been quite often excluded from the nonlinear predictive control application area.

\section{Simulations}

This section is devoted to some illustrative simulations, for the two systems already presented.

\subsection{Application 1 : the ball and beam system}

The dynamic model (16) of the ball and beam is considered with the parameters summarized in table 1. The choice of the scalar optimization parameter $p$ follows roughly the same ideas as for the preceding example. Namely, the parameterized set of admissible trajectories are chosen to be the least degree's polynomials satisfying the following conditions for a given initial pair $\xi^{0}=\left(\theta^{0}, \dot{\theta}^{0}\right)$

$$
T\left(\xi^{0}, p, 0\right)=\theta^{0} \quad ; \quad T\left(\xi^{0}, p, \tau_{c}\right)=\theta^{f} \quad ; \quad \dot{T}\left(\xi^{0}, p, 0\right)=\dot{\theta}^{0} \quad ; \quad \dot{T}\left(\xi^{0}, p, \tau_{c}\right)=\dot{\theta}^{f} \quad ; \quad T\left(\xi^{0}, p, \frac{\tau_{c}}{2}\right)=p
$$

therefore, here again, $p$ is chosen to be the value of $\theta$ in the middle of the sampling period. The final values $\theta^{f}$ and $\dot{\theta}^{f}$ depends on the control objective (the final target). The weighting matrix $Q$ is given by $Q=\operatorname{Diag}(1,0.2)$, and the switching time is $\tau_{c}=0.85$.

\subsubsection{Application 1: stabilization}

For stabilization at the equilibrium point, one takes $\xi^{f}=\left(\theta^{f}, \dot{\theta}^{f}\right)=0$ and $\eta^{f}=\left(r^{f}, \dot{r}^{f}\right)=0$. Figure 3.(a) enables a graphical verification of the stability sufficient conditions of point 1 . of proposition 1 .

Indeed, one may easily check that $(34)$ is satisfied for the following pairs of values $\left(k_{0}, \rho\right)_{1}=(2,0.28)$; $\left(k_{0}, \rho\right)_{2}=(3,0.42)$ while it is not satisfied for $k_{0}=1$. Figures 3.(b), 4.(a) show the evolution of $\xi$ and $\eta$, the control input versus time is plotted in figure 4.(b) as well as the phase portrait of $\eta$.

\subsubsection{Application 1: stabilization under parametric uncertainties}

Consider now the same model of the ball and beam, but this time under uncertainties on the inertias of the ball, as well as that of the beam, that is $J_{b}=J_{b} \pm \Delta J_{b} ; J=J \pm \Delta J$, the uncertainty on each inertia is of $50 \%$ of its corresponding nominal value. The behavior of $\xi$ and $\eta$ versus time is depicted in figures 5 . 


\subsubsection{Application 1: limit cycle generation}

In this case, the following non steady state desired final values are used $\xi^{f}=\left(\theta^{f}, \dot{\theta}^{f}\right)^{T}=\left(20^{\circ} 10^{\circ} / s\right)^{T}$ $; \eta_{f}=0$. Again, stability analysis can be investigated thanks to figure 6.(a), where one can easily check that the sufficient conditions given in point 2 of proposition 1 hold with the following values $\left(k_{0}, \rho, \varepsilon\right)=$ $(3,0.67,0.028)$. Figures 6.(b), 7.(a) show the evolution of $\xi$ and $\eta$ versus time, whereas figure 7.(b) displays the control input and the resulting phase portrait of $\eta$.

\subsubsection{Application 1: switching between limit cycles of different sizes}

The idea behind this application is to show the possibility of switching between different limit cycles. This may be done by applying steps on desired final configuration. Note that simultaneous changes in $\xi^{f}$ and $\eta^{f}$ may be done. For simplicity, Consider that only $\xi$ is subject to the following sequence $\left(\eta^{f}\right.$ remain unchanged)

- $\xi^{f}=\left(+20^{\circ}+10^{\circ} / s\right)$ for $\quad k=1 \ldots 10$

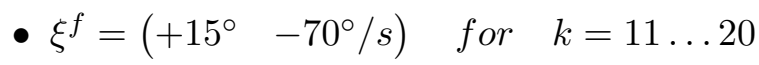

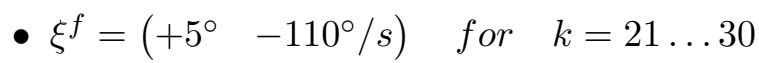

The resulting phase portrait is depicted on figure 8, as well as the corresponding control input.

\subsection{Application 2 : The modified impulsive Lorenz chaotic dynamical system}

Let us consider the impulsive Lorenz system given by (18)-(19) with $\mu=-0.6 ; \tau_{c}=0.2$ The origin is an equilibrium point unstable for the chaotic dynamical system (as discussed in [18]). The optimization parameter $p=x_{2}\left(\tau_{c} / 2\right)$ is considered. The weighting matrix is given by $Q=\operatorname{Diag}(1,1)$.

\subsubsection{Application 2 : stabilization}

For the purpose of stabilizing this impulsive system at the equilibrium $x=0$, consider the following target

state $\xi^{f}=x_{2}^{f}=0 \quad ; \quad \dot{x}_{2}^{f}=0 \quad ; \quad \eta^{f}=\left(\begin{array}{ll}x_{1}^{f} & x_{3}^{f}\end{array}\right)^{T}=\left(\begin{array}{ll}0 & 0\end{array}\right)^{T}$

In figure 9 a graphical plot of the map $\Psi_{k_{0}}^{Q}$ is depicted. Where a verification of the stability sufficient condition introduced by the point 1 . of proposition 1 could be done. We conclude therefore that the point 1. of proposition 1 is satisfied with $\left(k_{0}, \rho\right)=(1, \infty)$. We conclude that the closed-loop system trajectories converge globally to the equilibrium point. Figure 10 shows the system states versus time, as well as the control input and the phase portrait of $\eta$.

Three relatively large initial conditions have been considered, for which the objective is always to stabilize the system at the equilibrium position. The corresponding closed loop behavior as well as the input control and the phase portrait of $\eta$ are shown in figure 11.

\subsubsection{Application 2: limit cycle generation}

To investigate the limit cycle generation, consider the following state target $x_{2}^{f}=15, \eta^{f}=\left(\begin{array}{ll}0 & 0\end{array}\right)^{T}$. The stability analysis for the resulting closed-loop system is based on proposition 1 and the associated graphical tool. In figure 12 , the multi-step map $\Psi_{k_{0}}^{Q}\left(\right.$ for $k_{0}=1$ ) is depicted from which it may be clearly deduced that the conditions of point 2 . of proposition 1 hold for the following triplets of value $\left(k_{0}, \rho, \epsilon\right)=(1, \infty, 2.48)$. The system states versus time, as well as the control input, and the phase portrait of $\eta$ are displayed in figure 13 .

\subsection{Optimisation and computation time issues}

In the sequel some details concerning the optimization procedure, as well as the computation time will be introduced. 


\subsubsection{Optimization}

Note that the problem of optimization is beyond of the scope of this short paper, nevertheless, let us introduce some specifications on the optimization procedure.

- The main open-loop optimization problem, related to the control approach is that specified in (30), it is to be resolved on-line, and it uses the prediction of $\eta$ just before the next jump, this makes the control approach falling in the class of predictive control schemes.

- Only scalar optimization parameters have been considered, making significantly easier real time implementation of the proposed control scheme.

- On-line resolution of the optimization problem is carried out using the function DUVMIF, one of the Digital Fortran IMSL library optimization functions, which consists in finding the minimum point of a smooth function of a single variable using only function evaluations.

\subsubsection{Computation time}

The aim of this section is to make an evaluation of the computing time of the proposed control approach. The obtained results are then summarized in table 2, they concerns the case of limit cycle generation for both examples : ball and beam, and the modified Lorenz chaotic dynamical system, the computing time is essentially that of the resolution of the optimization problem, it is assessed on a computer equipped with a $2.5 \mathrm{Ghz}$ Intel microprocessor (the units of time in table 2 are seconds).

\begin{tabular}{|c||c|c|c|c|c|c|c|}
\hline & cycle 1 & cycle 2 & cycle 3 & cycle 4 & cycle 5 & cycle 6 & average \\
\hline \hline Ball \& Beam & 0.10 & 0.07 & 0.06 & 0.06 & 0.06 & 0.06 & 0.0683 \\
\hline Lorenz & 0.02 & 0.039 & 0.03 & 0.02 & 0.02 & 0.019 & 0.0247 \\
\hline
\end{tabular}

Table 2: Computing time evaluation

The average computing time to resolve numerically the optimization problem (30), leading to $p$ is about $68 \mathrm{msec}$ for the ball and beam system, and about $25 \mathrm{msec}$ for the modified Lorenz chaotic dynamical system.

\section{Conclusion \& future work}

In this paper, a new low dimensional predictive control scheme is proposed for the stabilization and stable limit cycle generation for a class of hybrid nonlinear systems. Computable sufficient conditions for the stability of the resulting closed-loop system are established based on the Poincaré section tool. These sufficient conditions can be easily viewed on $2 \mathrm{D}$ plots through off-line optimization. The results have been illustrated on two systems : the ball and beam (a classical jump free system), and the modified Lorenz impulsive chaotic system.

Several still unexplored questions are still to be answered. In particular, the way the control design parameters $\left(\xi^{f}, \eta^{f}, Q, \tau_{c}, \ldots\right)$ may be optimized in order to enhance some desired features such as robustness and cost reduction.

\section{References}

[1] M. Alamir and G. Bornard. On the stability of receding horizon control of nonlinear discrete-time systems. Systems \& Control Letters, 23:291-296, 1994.

[2] M. Alamir and N. Marchand. Constrained minimum-time oriented feedback control for the srabilization of nonholonomic systems in chained form. Journal of Optimization Theory and Applications, 118(2):229-244, 2003.

[3] H. Chen and F. Allgower. A quasi-infinite horizon nonlinear model predictive control scheme with guaranteed stability. Automatica, 34, No 10:1205-1217, 1998. 
[4] J. Hauser, S. Sastry, and P. kokotovic. Nonlinear control via approximate input-output linearisation : the ball and beam example. IEEE Trans. on Automat. Contr., 37(3):392-398, 1992.

[5] A. Isidori. Nonlinear Control Systems. Springer Verlag, 1989.

[6] S. S. Keerthi and E. G. Gilbert. Optimal infinite horizon feedback laws for a general class of constrained discrete time systems. J. Optim. Theory Applics, , 57:265-293, 1988.

[7] L. Magni, G. De Nicolao, and R. Scattolini. Output feedback receding horizon control of discrete-time nonlinear systems. In Proceedings of the 4th IFAC Nonlinear Control Systems Design Symposium, pages 422-427, Oxford, UK, 1998.

[8] L. Magni and R. Sepulchre. Stability margins of nonlinear receding horizon control via inverse optimalty. Systems $\&$ Control Letters, 32(4):241-245, 1998.

[9] N. Marchand and M. Alamir. Numerical stabilization of a rigid spacecraft wih two actuators. Journal of Dynamic Systems Measurement and Control, 125(3):489-491, 2003.

[10] D. Q. Mayne and H. Michalska. Receding horizon control of nonlinear systems. IEEE Trans. on Automatic Control, 35:814-824, 1990.

[11] D. Q. Mayne, J. B. Rawlings, C. V. Rao, and P. O. Scokaert. Constrained model predictive control: Stability and optimality. Automatica, 36:789-814, 2000.

[12] E. S. Meadows, M. A. Henson, J. W. Eaton, and J. B. Rawlings. Receding horizon control and discontinuous state feedback stabilization. International Journal of Control, 62(5):1217-1229, 1995.

[13] H. Michalska. A new formulation of receding horizon stabilizaing control without terminal constraint on the state. European Journal of Control, 3(1):2-14, 1997.

[14] G. De Nicolao, L. Magni, and R. Scattolini. On the robustness of receding-horizon control with terminal constraints. IEEE Trans. Automatic Control, 41(3):451-453, 1996.

[15] T. Ohtsuka and H. Fujii. Real-time optimization algorithm for nonlinear receding horizon control. Automatica, 33(6):1147-1154, 1997.

[16] T. Parisini and S. Sacone. A hybrid receding horizon control scheme for nonlinear systems. Systems 86 Control Letters, 38, No 3:187-196, 1999.

[17] E. R. Westervelt, J. W. Grizzle, and D. E. Koditschek. Hybrid zero dynamics of planar biped walkers. IEEE Trans. on Automat. Contr., 48(1):42-56, 2003.

[18] T. Yang. Impulsive control theory. Lecture Notes in Control and Information Sciences. Springer Verlag, Berlin, Germany, 2001. 
Application 1 : The ball and beam system

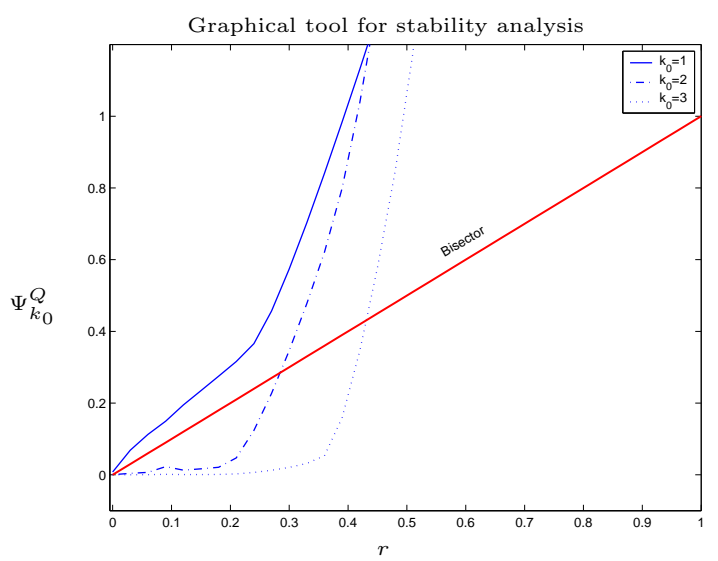

(a)
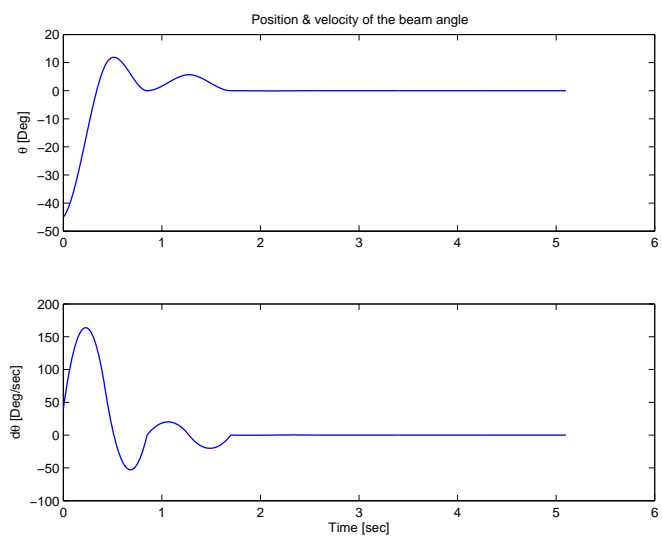

(b)

Figure 3: Application 1 (Stabilization) : graphical stability analysis curves (a), $\xi$ versus time (b).
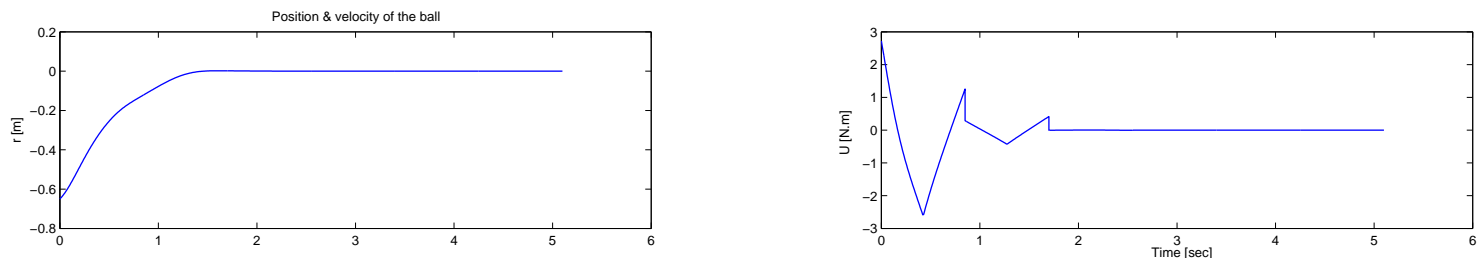

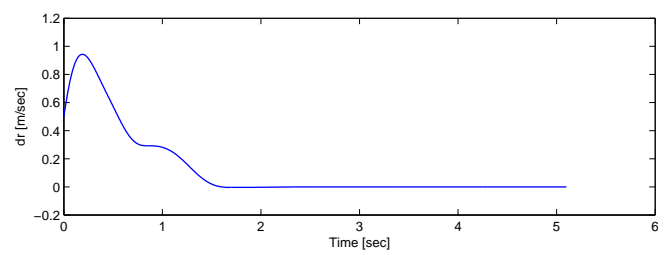

(a)

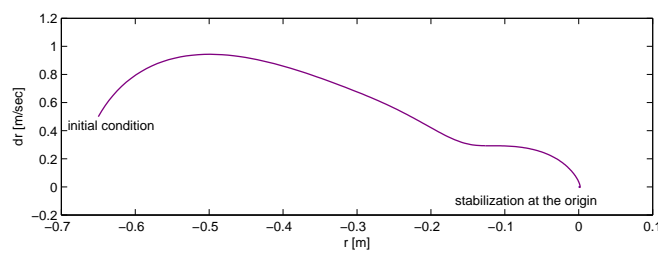

(b)

Figure 4: Application 1 (Stabilization) : $\eta$ versus time (a), control input (b)top, $\eta$ phase portrait (b)bottom.
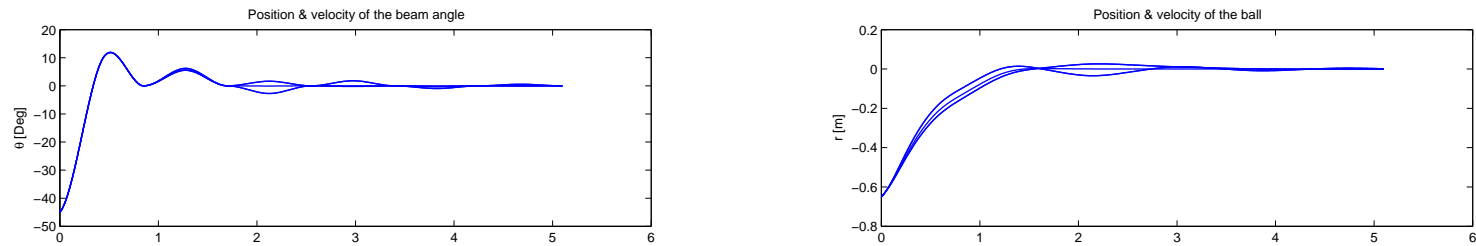

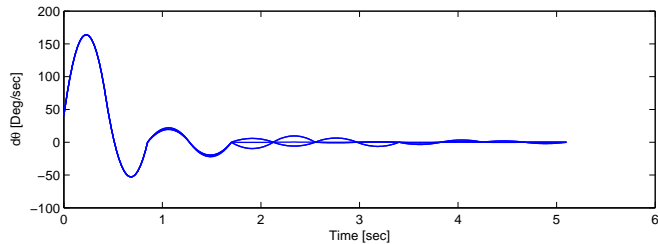

(a)

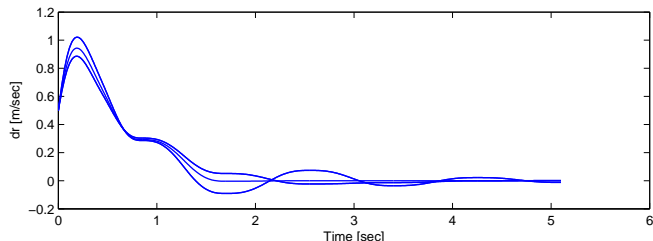

(b)

Figure 5: Application 1 (stabilization of the uncertain system) : $\xi$ versus time (a), $\eta$ versus time (b). 


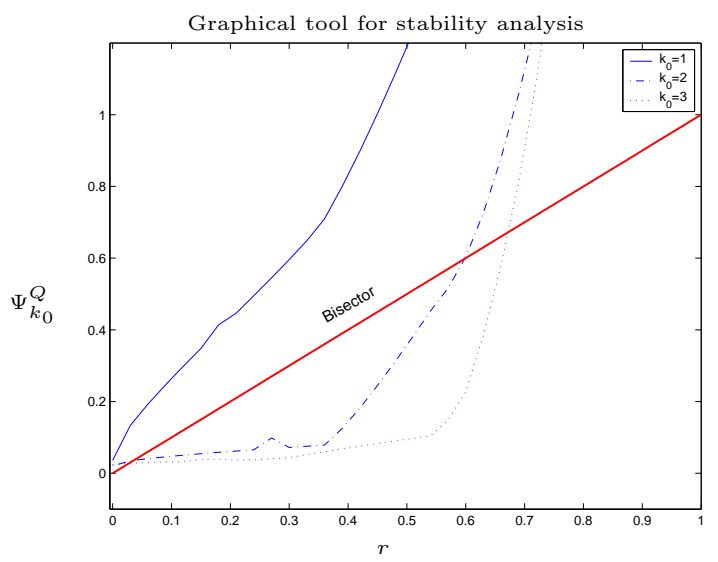

(a)
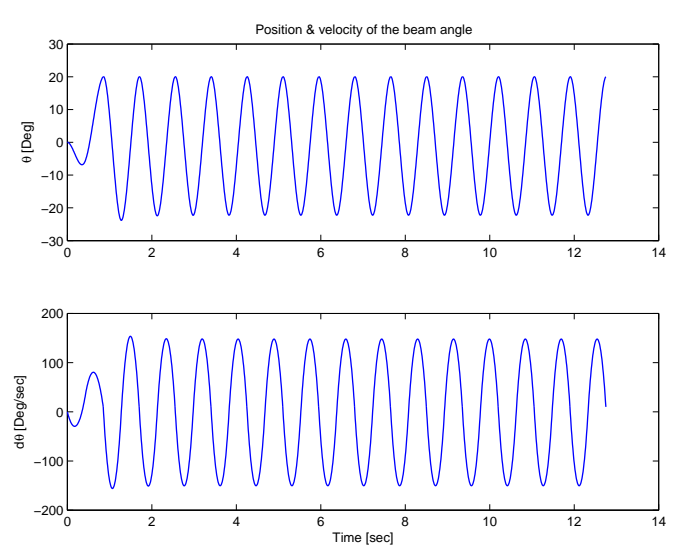

(b)

Figure 6: Application 1 (limit cycle generation) : graphical stability analysis curves (a), $\xi$ versus time (b).
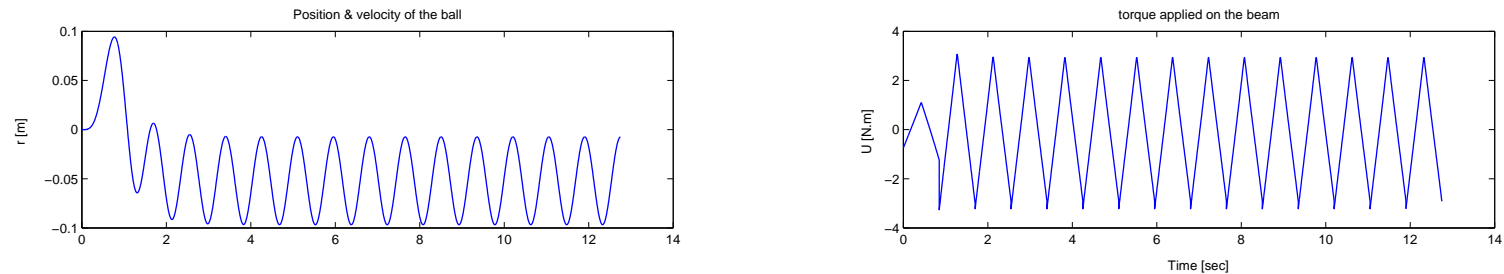

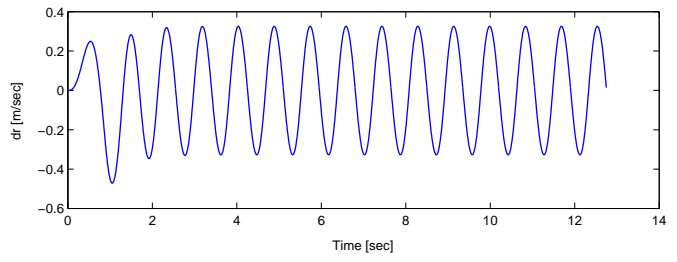

(a)

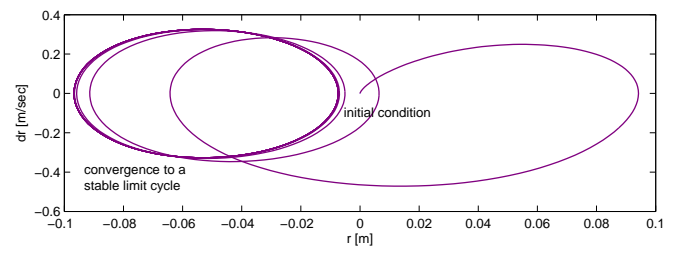

(b)

Figure 7: Application 1 (limit cycle generation) : $\eta$ versus time (a), control input (b)top, $\eta$ phase portrait (b)bottom.

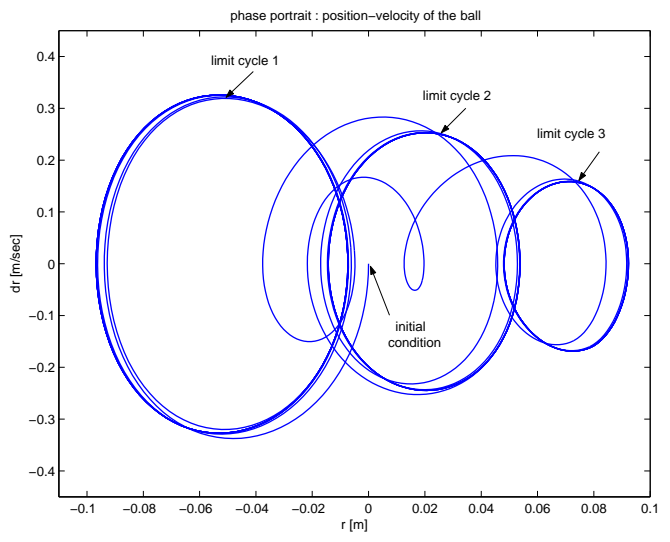

(a)

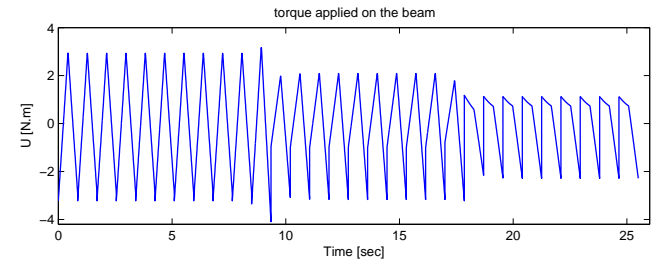

(b)

Figure 8: Application 1 (limit cycle generation) : switching between limit cycles (a), corresponding control input (b). 
Application 2 : The modified impulsive Lorenz chaotic dynamical system

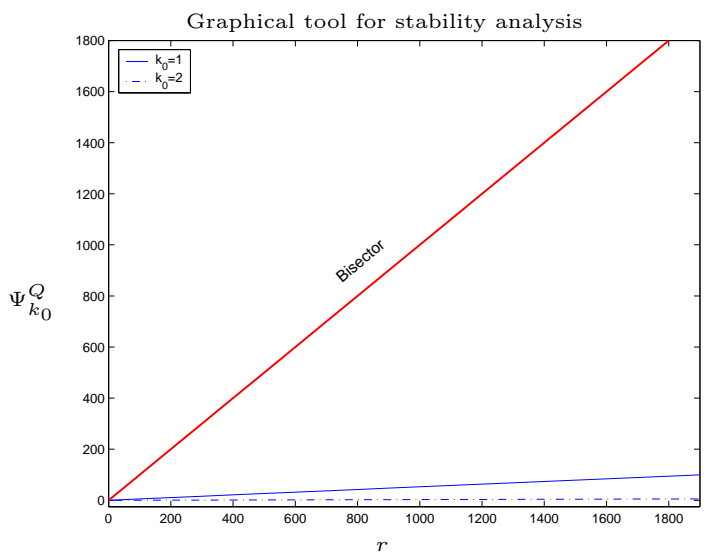

Figure 9: Application 2 (Stabilization) : graphical stability analysis curves.
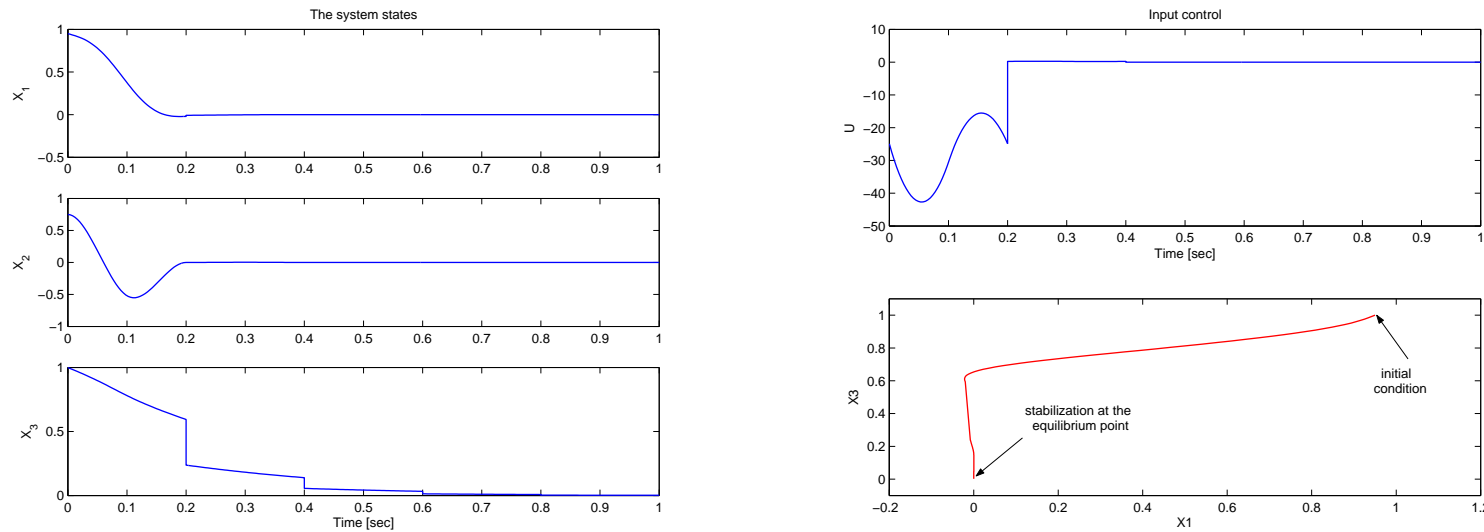

(a)

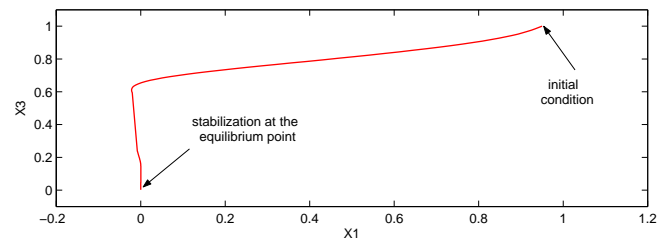

(b)

Figure 10: Application 2 (Stabilization) : states versus time (a). control input (b)top, $\eta$ phase portrait (b)bottom.
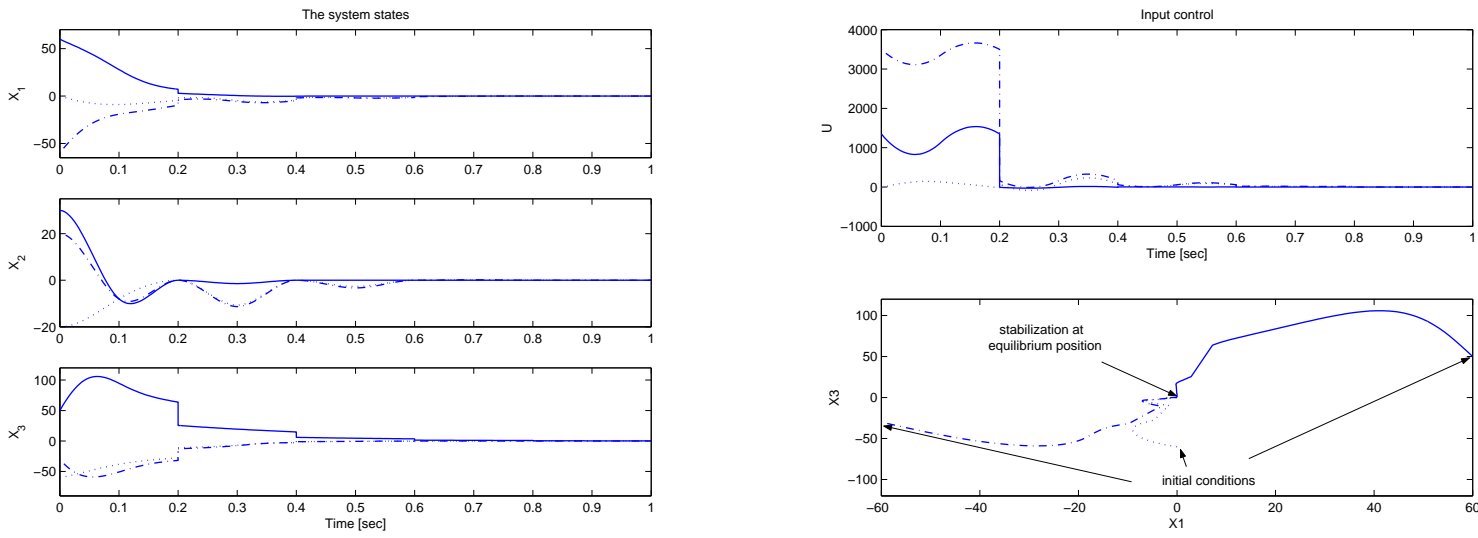

(a)

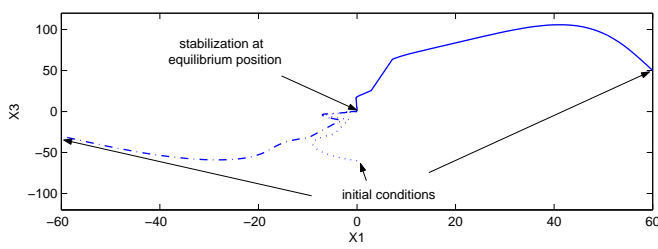

(b)

Figure 11: Application 2 (stabilization) : states versus time (a), control input (b)top, $\eta$ phase portrait (b)bottom. 


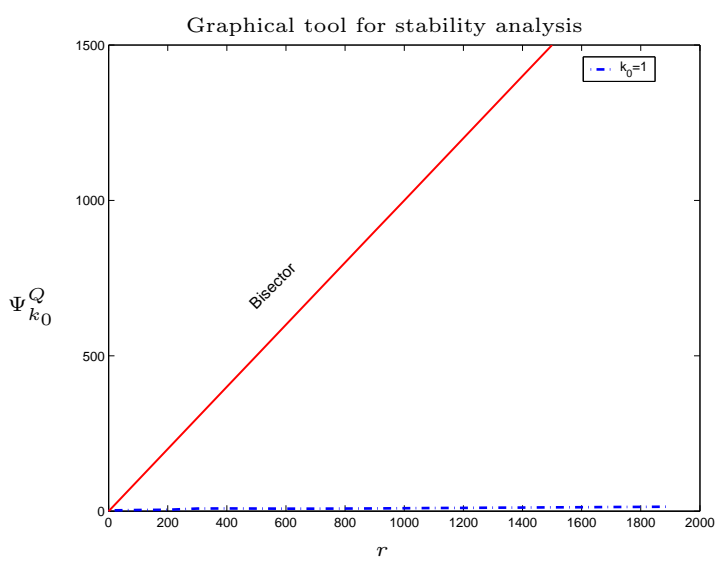

Figure 12: Application 2 (limit cycle generation) : graphical stability analysis curves.



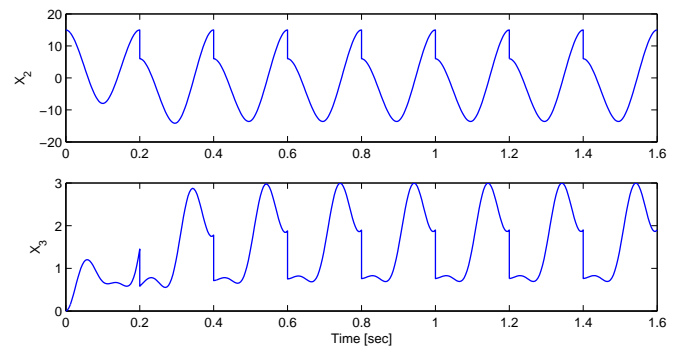

(a)
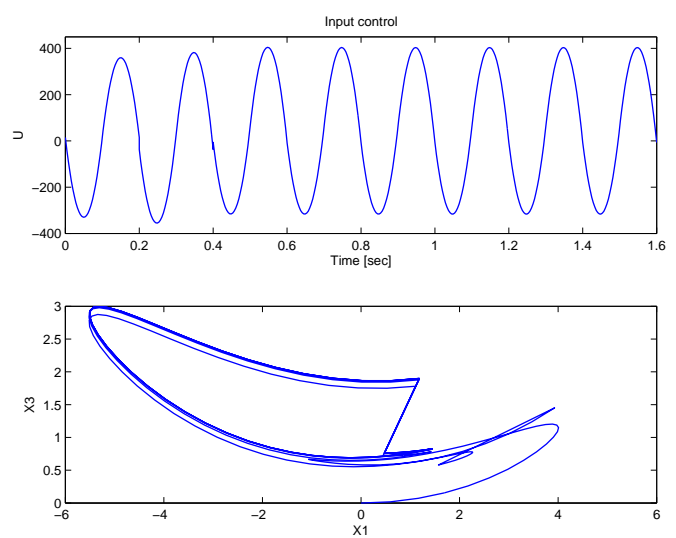

(b)

Figure 13: Application 2 (limit cycle generation) : states versus time (a), control input (b)top, $\eta$ phase portrait (b)bottom. 